\title{
Best Fit of Mixture For Distributed Poisson Multi-Bernoulli Mixture Filtering
}

\author{
Tiancheng Li and Kai Da
}

\begin{abstract}
The Poisson multi-Bernoulli mixture (PMBM) filter is extended for distributed implementation using a wireless sensor network. At the core of the proposed networking approach, the PMBM posterior is decomposed into two parts corresponding to the undetected and detected targets, respectively. Fusion is motivated to be performed with regard to the latter only which is represented by MBM based on a distributed flooding algorithm for internode communication, which iteratively shares the MBMs between neighbor sensors. Then, a suboptimal "best-fit-ofmixture" principle is followed at each local sensor to find a MBM that best fits the mixture of MBMs aggregated from distinct sensors, leading to an arithmetic average (AA) of these MBMs. We prove the exact closure of the MBM-AA fusion and discuss its sub-optimality and limitations. Simulation demonstrates the effectiveness and limitations of our approach.
\end{abstract}

Index Terms-Target tracking, multisensor fusion, arithmetic average, Poisson multi-Bernoulli mixture, distributed flooding.

\section{INTRODUCTION}

D ISTRIBUTED multitarget detection and tracking (DMTDT) using a wireless sensor network in clutter and noises arises in a variety of application domains such as air traffic control, surveillance, oceanography, remote sensing and biomedical research, to name a few [1]-[3]. Random finite set (RFS) has identified a natural and promising tool for modeling MTDT in the Markov-Bayes optimal paradigm [4][6]. However, a key challenge arises from the complicated cross-correlation between netted sensors, which is typically unknown and refuses the Bayes optimal fusion. Fortunately, it was found that both linear/arithmetic average (AA) and geometric/log-linear average (GA) fusion approaches are qualified to solve this problem [7]-[9]. Average fusion is efficient in computation and tolerant to sensor fault/failure and therefore satisfies a key requirement of the realistic distributed sensor network which typically comprises low-cost, batterypowered sensor nodes with limited capacities for computing, communicating and sensing.

Recently, various RFS filters have been extended for efficient, distributed multi-sensor implementation based on AA fusion, including PHD filters based on Gaussian mixture (GM)

Manuscript submitted on 21 May 2020

Preprint DOI: $10.36227 /$ techrxiv. 12351710

This work was partially supported by Key Laboratory Foundation of National Defence Technology under Grant 61424010306, by Space Science and Technology and by Fundamental Research Funds for the Central Universities.

$\mathrm{T}$. Li is with the Key Lab of Information Fusion Technology (Ministry of Education), School of Automation, Northwestern Polytechnical University, Xi'an 710129, China, e-mail: t.c.li@nwpu.edu.cn

K. Da is with the National Key Laboratory of Science and Technology on ATR, National University of Defense Technology, Changsha 410073, China, e-mail: dktm131@163.com implementation [10], [11] or particle implementation [12][14], cardinalized PHD filters for standard models [15], [16] or for jump Markov system [17], Bernoulli filter [18], multiBernoulli (MB) filter [9] and labelled RFS filters [19]. Prior to these, GA-fusion-based RFS filters have been a research focus of the community which are more commonly known as generalized covariance intersection [20]-[26] and geometric/exponential mixture density [27], [28], to name a few. Both AA and GA fusion rules have demonstrated great potential for multi-target information fusion.

Different from the (C)PHD and MB filters, the Poisson multi-Bernoulli mixture (PMBM) filter [29]-[31] has a closedform filtering recursion (namely closure) based on standard state space models with Poisson birth and has demonstrated better performance [32], [33] when the target detection probability is low. That is, the PMBM is a multi-target conjugate prior. Another relevant conjugate prior in the RFS family is the generalized labelled MB [6], [34], [35]. The PMBM consists of the combination of a Poisson point process (PPP) and a multi-Bernoulli mixture (MBM), where the PPP represents all undetected targets which enables the filter more sensitive to target birth while the MBM considers different track-measurement-association hypotheses gaining a higher accuracy than a single MB. The success of the PMBM filter gives rise to three variants: 1) If the birth model is $\mathrm{MB}$ or MBM instead of PPP, the PMBM filter reduces to a MBM filter [31], [36]. 2) If only one global data association hypothesis is maintained in the MBM, the PMBM filter reduces to a PMB filter [32], [37]. 3) By extending to the continuous time multitarget system, the PMBM filter is extended to a continuousdiscrete PMBM filter [38].

Recently, the GA fusion has been exploited for PMB fusion in [39], [40], which fuses the PPP and MB separately and approximately by assuming all targets well spaced. PMBM fusion can be addressed similarly with regard to the PPP and MBM, respectively. However, the MBM-GA fusion does not admit exact closure and so far it is even unclear how to approximate it.

In this work, we investigate the distributed flooding algorith$\mathrm{m}$ [41] for internode MBM communication which iteratively exchanges the MBMs between neighbor sensors, resulting in a mixture of MBMs at each sensor. Then, following the "best-fit-of-mixture" fusion principle, a MBM is found that fits the mixture of MBMs with minimum KLD and 2-norm distance, which is exactly given by the AA of these MBMs. We prove this exact closure for MBM-AA fusion. Moreover, for better communication and computation efficiency, only a single or a few MBs in the MBM of each individual sensor 
are disseminated to the other sensors, while the PPP that is typically of minor intensity and does not admit closure for averaging fusion remains unchanged at individual sensors.

This paper is organized as follows. Preliminaries regarding the standard model we consider, MB, and MBM are given in Section II. Optimal multi-sensor fusion, sub-optimality of the AA fusion and the idea of best-fit-of-mixture are analyzed and illustrated in Section III. The exact closure of the MBM-AA fusion and its implementation via MBM-flooding are given in Section IV. Simulation results are given in Section V. We conclude in Section VI.

\section{PRELIMINARIES}

\section{A. Standard Models}

The realization of an RFS of the multitarget states is a set $\mathbf{X}=\left\{\mathbf{x}_{1}, \mathbf{x}_{2}, \ldots, \mathbf{x}_{n}\right\}$, where $n=|\mathbf{X}|$ is the random number of targets, and $\mathbf{x}_{i} \in \mathbb{R}^{d}$ is the state vector of the $i$-th target. The random nature of the multitarget set $\mathbf{X}$ is captured by its probability density, denoted by $f(\mathbf{X})$. For any realization $\mathbf{X}=\left\{\mathbf{x}_{1}, \mathbf{x}_{2}, \ldots, \mathbf{x}_{n}\right\}$ with a given cardinality $|\mathbf{X}|=n$,

$$
f(\mathbf{X})=n ! \rho(n) f_{n}\left(\mathbf{x}_{1}, \ldots, \mathbf{x}_{n}\right),
$$

where, the cardinality distribution is $\rho(n) \triangleq \operatorname{Pr}\{|\mathbf{X}|=n\}$.

Considering a sensor network composed of sensors $s=$ $1,2, \ldots, S$, we denote by $\mathcal{S}_{s}$ the set of neighbor sensors of sensor $s$. We assume that the fields of view of all sensors are identical, in which there are a random, time-varying number of targets. Each sensor operates a PMBM filter [29]-[31] for detecting and tracking these targets. We note that these sensors are typically correlated in the information they have about the targets and in their prior knowledge, in an unknown manner.

Targets arrive at each time according to a non-homogeneous PPP, independent of target survivals. Setting the the cardinality of new-born target RFS as a Poisson distribution with rate $\lambda$ (namely $\rho(n)=e^{-\lambda} \lambda^{n} / n$ !) in (1) yields a multidimensional Poisson distribution [4, p.366],

$$
f\left(\mathbf{X}^{\mathrm{p}}\right)=e^{\lambda} \lambda^{n} \prod_{i=1}^{n} p\left(\mathbf{x}_{i}\right) .
$$

Hereafter, $p(\mathbf{x})$ is a probability density function (PDF) of single target.

Each target evolves and is measured by each sensor independent of the other targets. The surviving process of each target is Bernoulli. That is, at time $k-1$, the target with state $\mathbf{x}_{k-1}$ will either die with probability $1-p_{k}^{\mathrm{s}}$ or persists at time $k$ with survival probability $p_{k}^{\mathrm{s}}$ and attains a new state $\mathbf{x}_{k}$ according to a Markov jump PDF $f_{k \mid k-1}\left(\mathbf{x}_{k} \mid \mathbf{x}_{k-1}\right)$.

Given a target with state $\mathbf{x}_{k}$, sensor $s$ either detects it with probability $p_{s, k}^{\mathrm{d}}$ and generates a measurement $\mathbf{z}_{s, k} \in \mathbf{Z}_{s, k}$ with likelihood $g_{s, k}\left(\mathbf{z}_{s, k} \mid \mathbf{x}_{k}\right)$ or fails to detect it with probability $1-p_{s, k}^{\mathrm{d}}$, where $\mathbf{Z}_{s, k}$ denotes the set of measurements received at time $k$ by sensor $s$. The clutter at sensor $s$ follows a Poisson RFS as in (2) with Poisson rate $\kappa_{s}$, independent of real measurements of targets.

\section{B. Bernoulli RFSs and Their Union: MB}

A Bernoulli RFS can either be empty (with probability $1-r$ ) or have one element (with probability $r$ ), distributed over the state space according to $\operatorname{PDF} p(\mathbf{x})$. That is, the probability distribution of the Bernoulli RFS $\mathbf{X}^{\mathrm{b}}$ is

$$
f\left(\mathbf{X}^{\mathrm{b}}\right)= \begin{cases}1-r, & \text { if } \mathbf{X}=\emptyset \\ r p(\mathbf{x}), & \text { if } \mathbf{X}=\{\mathbf{x}\} \\ 0, & \text { otherwise }\end{cases}
$$

To represent the posterior of a random number of (no more than $M$ ) targets, $M$ Bernoulli RFSs $\mathbf{X}^{\mathrm{b}}$ with respective target existence probabilities $r^{(i)}$ and state PDFs $p^{(i)}(\cdot), i=$ $1,2, \ldots, M$, can be used. Their linear union is a MB RFS

$$
\mathbf{X}^{\mathrm{mb}}=\bigcup_{\ell=1}^{M} \mathbf{X}_{\ell}^{\mathrm{b}},
$$

which is completely characterized by $M$ parameter pairs $\left\{r^{(i)}, p^{(i)}(\cdot)\right\}_{i=1}^{M}$. The corresponding MB distribution can be expressed, for any given cardinality $\left|\mathbf{X}^{\mathrm{mb}}\right|=n$, as follows

$$
f\left(\mathbf{X}^{\mathrm{mb}}\right)=\sum_{\mathbf{X}_{1}^{\mathrm{b}} \sqcup \cdots \sqcup \mathbf{X}_{n}^{\mathrm{b}}=\mathbf{X}^{\mathrm{mb}}} \prod_{i=1}^{n} f_{i}\left(\mathbf{X}^{\mathrm{b}}\right) .
$$

where $\sqcup$ stands for disjoint union.

\section{Weighted Union of MBs: MBM [37]}

The MB can efficiently approximate the posterior multitarget density [42]. This approximation can be improved in accuracy by using a linear combination of multiple MBs, namely a MBM, where different MBs correspond to different global hypotheses of measurements-to-target association history. That is, the MBM RFS is a normalized and weighted sum of multitarget densities of MBs, which can be parameterized by a mixture of MB RFSs

$$
\left\{w_{j},\left\{r_{j}^{(i)}, p_{j}^{(i)}(\cdot)\right\}_{i \in \mathbb{I}_{j}}\right\}_{j \in \mathbb{J}},
$$

where $\mathbb{I}_{j}$ is the index set of the BCs in MB $j, \mathbb{J}$ is the index set of the MBs in the MBM (each term corresponding to a global hypothesis), and $w_{j} \geq 0$ is a coefficient/probability assigned to $\mathrm{MB} /$ hypothesis $j \in \mathbb{J}$, subject to

$$
\sum_{j \in \mathbb{J}} w_{j}=1
$$

For any MBM realization $\mathbf{X}^{\mathrm{mbm}}=\left\{\mathbf{x}_{1}, \ldots, \mathbf{x}_{n}\right\}$, the multi-target distribution is, c.f. (5)

$$
f\left(\mathbf{X}^{\mathrm{mbm}}\right) \propto \sum_{j \in \mathbb{J}} w_{j} \sum_{\mathbf{X}_{1}^{\mathrm{b}} \sqcup \cdots \sqcup \mathbf{X}_{n}^{\mathrm{b}}=\mathbf{X}^{\mathrm{mb}}} \prod_{i=1}^{n} f_{j, i}\left(\mathbf{X}^{\mathrm{b}}\right)
$$

where $\propto$ stands for proportionality.

Note that global hypotheses are made up of single hypotheses/BCs each of which corresponding to a potential target [29]. Instead of posing a weight for each global hypothesis, one may weight single hypothesis/BC $i$ in global hypothesis $j$ by $w_{j, i}$; see e.g., [31], [36]. Then, $w_{j} \propto \prod_{i=1}^{n} w_{j, i}$. 


\section{OPTIMAL AND SUB-OPTIMAL FUSION}

\section{A. Optimal Fusion and Conservative Fusion}

Consider two correlated posteriors $f\left(\mathbf{X} \mid \mathbf{Z}_{1,1: k}\right)$, $f\left(\mathbf{X} \mid \mathbf{Z}_{2,1: k}\right)$ at time $k$. Their joint posterior is given conceptually as follows [8], [43]:

$$
\begin{aligned}
f\left(\mathbf{X} \mid \mathbf{Z}_{1,1: k}, \mathbf{Z}_{2,1: k}\right) & =f\left(\mathbf{X} \mid \mathbf{Z}_{1,1: k} \cup \mathbf{Z}_{2,1: k}\right), \\
& \propto \frac{f\left(\mathbf{X} \mid \mathbf{Z}_{1,1: k}\right) f\left(\mathbf{X} \mid \mathbf{Z}_{2,1: k}\right)}{f\left(\mathbf{X} \mid \mathbf{Z}_{1,1: k} \cap \mathbf{Z}_{2,1: k}\right)},
\end{aligned}
$$

where the denominator in (9) is used to divide out the common information between the two fusion sources.

Netted sensors which observe the same targets often use the same prior information and model assumptions. Unfortunately, despite favorite, simple cases with a-priori information [44][47], it is practically intractable to measure the common information. Then, it becomes important to seek conservative fusion that avoids underestimating the actual squared estimate errors [8], [48]-[50]. That is, for a posterior $f_{s}(\mathbf{x})$ consisting of estimate mean $\hat{\mathbf{x}}_{s} \triangleq \int \mathbf{x} f_{s}(\mathbf{x}) d \mathbf{x}$ and error covariance matrix $\mathbf{P}_{s} \triangleq \int\left(\mathbf{x}-\hat{\mathbf{x}}_{s}\right)\left(\mathbf{x}-\hat{\mathbf{x}}_{s}\right)^{\mathrm{T}} f_{s}(\mathbf{x}) d \mathbf{x}$ regarding the real state $\mathbf{x}$, it is conservative if and only if $\mathbf{P}_{s}$ is no less than the actual mean square error of the estimate, i.e., $\mathbf{P}_{s}-\mathrm{E}\left[\left(\mathbf{x}-\hat{\mathbf{x}}_{s}\right)\left(\mathbf{x}-\hat{\mathbf{x}}_{s}\right)^{\mathrm{T}}\right]$ is positive semi-definite.

\section{B. Sub-optimality of AA Fusion}

As a proven conservative fusion rule [8], the AA fusion of probability distributions $f_{s}(\mathbf{X}), s=1,2, \cdots, S$ is given by

$$
f_{\mathrm{AA}}(\mathbf{X}) \triangleq \frac{1}{S} \sum_{s=1}^{S} f_{s}(\mathbf{X}) \text {. }
$$

Essentially, the AA is a Fréchet mean corresponding to the 2-norm distance based Fréchet function ${ }^{1}$ [9],

$$
f_{\mathrm{AA}}(\mathbf{X})=\underset{g}{\arg \min } \frac{1}{S} \sum_{s=1}^{S}\left\|f_{s}-g\right\|^{2},
$$

where $\|f\|^{2}=\int(f(\mathbf{X}))^{2} \delta \mathbf{X}$ is a set integral [4, Ch.11.3.3].

Relatively, the AA fusion also minimizes the average of the KLDs of the fused result with relative to fusing probability distributions $f_{s}(\mathbf{X}), s=1,2, \cdots, S$, [52]

$$
f_{\mathrm{AA}}(\mathbf{X})=\underset{g}{\arg \min } \frac{1}{S} \sum_{s=1}^{S} D_{\mathrm{KL}}\left(f_{s} \| g\right),
$$

where the KLD of the probability distribution $g(\mathbf{X})$ relative to $f(\mathbf{X})$ is given as $D_{\mathrm{KL}}(f \| g)=\int f(\mathbf{X}) \log \frac{f(\mathbf{X})}{g(\mathbf{X})} \delta \mathbf{X}$.

Mathematically, factor $1 / S$ can be dropped from both (11) and (12) without affecting the equation. Then, it becomes more evident that, the probability distribution that best fits the mixture of probability distributions $\left\{f_{s}\right\}_{s=1}^{S}$ in the sense of minimizing both the 2-norm distance and KLD is the AA of all terms in the mixture. Considering that the mixture of these posteriors $\left\{f_{s}\right\}_{s=1}^{S}$ contains the complete posterior

\footnotetext{
${ }^{1}$ Fréchet function corresponding to a Fréchet mean like AA may not be unique. For example, the Cauchy-Schwarz divergence reduces a 2-norm distance for PPP [51] and therefore the sum of it serves as another Fréchet function for the AA in the case of PPP RFS; see also [13].
}

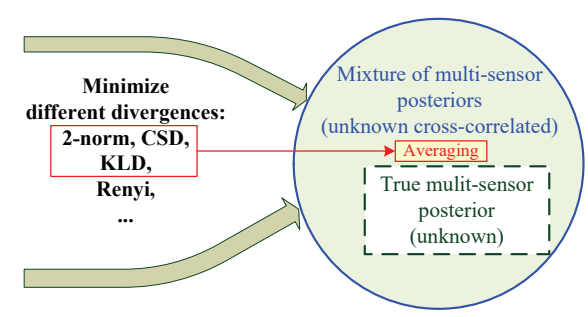

Fig. 1. Approach the true multi-sensor posterior by fitting the mixture of multiple sensor posteriors using various optimization distances.

information yielded by all sensors, it is a reasonable substitute of the multi-sensor true posterior. The mixture, however, contains common information of the sensors (as assumed from the beginning) and is no more than an approximate of the true posterior unless the common information are divided out properly. This essentially differs from the fit of the true posterior in e.g., [37], [53]. Therefore, the optimization as in both (11) and (12) is suboptimal.

The principle of best-fit-of-mixture can be illustrated as in Fig. 1. In fact, what has been done with AA-PHD fusion [10][14], AA-CPHD fusion [15]-[17], BC-AA fusion [18], MBAA fusion [9] and RFS-GA fusion [20]-[28] all essentially follow the best-fit-of-mixture principle, aiming to best fit the mixture of unknown-correlated PHDs, CPHDs, BCs and MBs from different sensors, respectively. The key challenge of the fit, however, is from non-closure, for example, the AA of $\mathrm{PPPs} / \mathrm{MBs}$ is no longer a PPP/MB.

\section{Distributed Flooding-BASEd MBM-AA Fusion}

\section{A. PMBM Conjugate Prior}

Based on standard state space model assumptions with Poisson target birth, the PMBM conjugate prior at sensor $s$ is given by [29], [37]

$$
f_{s}\left(\mathbf{X}^{\mathrm{pmbm}}\right)=\sum_{\mathbf{X}^{\mathrm{p}} \sqcup \mathbf{X}^{\mathrm{mbm}}=\mathbf{X}^{\mathrm{pmbm}}} f_{s}\left(\mathbf{X}^{\mathrm{p}}\right) f_{s}\left(\mathbf{X}^{\mathrm{mbm}}\right) .
$$

As shown, the PMBM consists of a PPP component and a MB$\mathrm{M}$ component, which represent the undetected targets (which are hypothesised to exist but have never been detected) and the detected targets, respectively [29]. Details for predicting and updating both PPP and MBM are provided in [29], [31], which are omitted here.

Continuously-missed detections that contribute to the PPP are various at different sensors due to the independent, random nature of the detecting event. Furthermore, the PPP usually has a minor intensity and does not admit closure for averaging. Therefore, there is little need but significant difficulty to fuse the PPPs. Following this line of thinking, we do not perform fusion over the PPPs obtained at different sensors. Only the MBMs are communicated and fused over the sensor network. This also saves communication and fusion calculation.

The proposed multi-sensor PMBM filter can be illustrated in Fig. 2. As to be addressed next, both flooding and AA fusion of the MBMs need no approximation, maintaining an exact MBM structure. Clearly, these approaches are straightforwardly applicable to the MBM filter [31], [36]. 


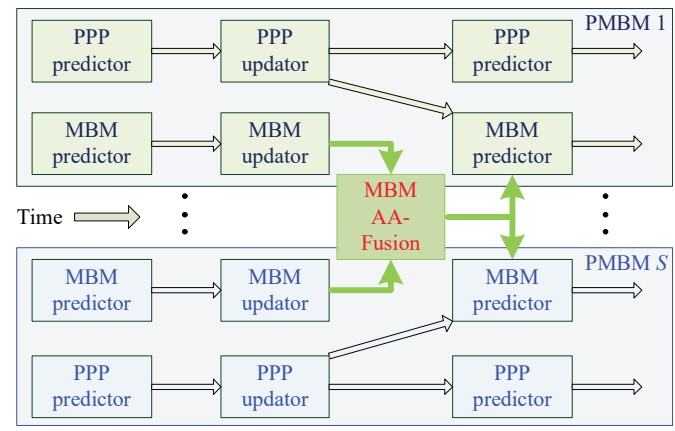

Fig. 2. Algorithm flow of proposed distributed PMBM filter.

\section{B. $A A$ of $M B M s$}

The following Lemma lies at the core of our proposed fusion approach.

Lemma 1. Given $S M B M$ with multi-target probability distribution as follows, $s=1,2, \ldots, S$

$$
f_{s}\left(\mathbf{X}^{\mathrm{mbm}}\right) \propto \sum_{j \in \mathbb{J}_{s}} w_{s, j} \sum_{\mathbf{X}_{1}^{\mathrm{b}} \sqcup \cdots \sqcup \mathbf{X}_{n}^{\mathrm{b}}=\mathbf{X}^{\mathrm{mb}}} \prod_{i=1}^{n} f_{s, j, i}\left(\mathbf{X}^{\mathrm{b}}\right),
$$

their AA given by

$$
f_{\mathrm{AA}}\left(\mathbf{X}^{\mathrm{mbm}}\right)=\frac{1}{S} \sum_{s=1}^{S} f_{s}\left(\mathbf{X}^{\mathrm{mbm}}\right),
$$

remains a MBM.

Proof. The proof is straightforwardly given by substituting (14) in (15), which yields a MBM as shown in (16)

$$
\begin{aligned}
f_{\mathrm{AA}}(\mathbf{X}) & \propto \frac{1}{S} \sum_{s=1}^{S} \sum_{j \in \mathbb{J}_{s}} w_{s, j} \sum_{\mathbf{X}_{1}^{\mathrm{b}} \sqcup \ldots \sqcup \mathbf{X}_{n}^{\mathrm{b}}=\mathbf{X}^{\mathrm{mb}}} \prod_{i=1}^{n} f_{s, j, i}\left(\mathbf{X}^{\mathrm{b}}\right), \\
& =\sum_{j \in \mathbb{J}_{1: S}} \tilde{w}_{j} \underbrace{\sum_{\mathbf{X}_{1}^{\mathrm{b}} \sqcup \cdots \sqcup \mathbf{X}_{n}^{\mathrm{b}}=\mathbf{X}^{\mathrm{mb}}} \prod_{i=1}^{\prod_{\tilde{s}}(j), j, i}\left(\mathbf{X}^{\mathrm{b}}\right)}_{=f\left(\mathbf{X}^{\mathrm{mb}}\right), \text { c.f. (5) }},
\end{aligned}
$$

where

$$
\begin{gathered}
\tilde{w}_{j}=\frac{w_{s, j}}{S}, \\
\mathbb{J}_{1: S}=\bigcup_{s=1}^{S} \mathbb{J}_{s}, \\
\tilde{s}(j)=\left\{s \mid j \in \mathbb{J}_{s}\right\} .
\end{gathered}
$$

Lemma 1 indicates that the MBM-AA fusion admits exact closure. Key operations for such a fusion are mixing and reweighting the MBMs as shown in (17) and (18). Indeed, the (weighted) mixtures of multiple Bernoulli RFSs, MB RFSs and MBM RFSs are a MB RFS, MBM RFS and MBM RFS, resepctively, as illustrated in Fig. 3. In addition to MBM that maintains closure for mixture union operation, some other popular mixture distributions such as the Dirac delta mixture (commonly known as particle posterior) and GM also admit exact closure for AA fusion.

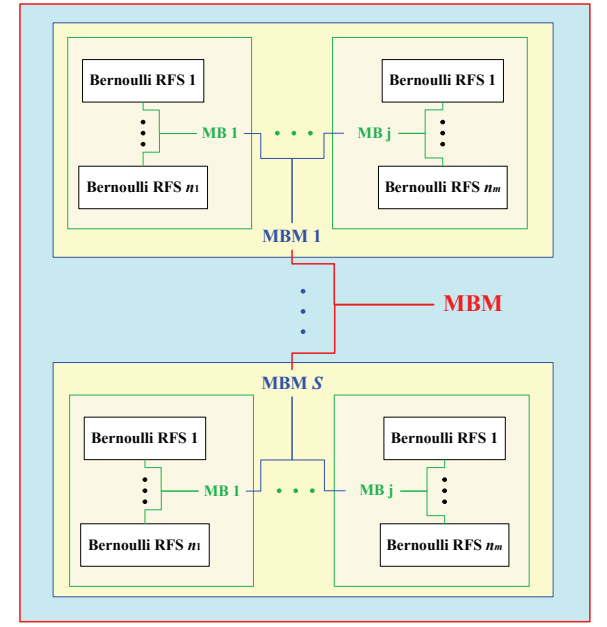

Fig. 3. The mixtures of multiple Bernoulli RFSs, MB RFSs and MBM RFSs are a MB RFS, a MBM RFS and a MBM RFS, resepctively.

We now address how the MBMs can be disseminated in a distributed manner that maintains exact closure. We consider the distributed flooding scheme [41] which is naturally consistent with the mixture union operation as shown in (18) and guarantees exact and efficient convergence.

\section{MBM Mixing by Flooding}

In distributed flooding [41], each local sensor serves equivalently like a fusion center which mixes the relevant information from the other sensors via iterative neighborhood communication. Re-weighting/scaling is carried out at the end of all communication iterations in each filtering step. For clarity, we explain the MBM-flooding algorithm here with respect to the global hypotheses corresponding to MBM distributions. That is, the flooding algorithm updates the set of global hypotheses of sensor $s$ at iteration $t=0,1, \cdots$ as follows

$$
\mathbb{J}_{s}(t+1)=\mathbb{J}_{s}(t) \cup \mathbb{U}_{s}^{\mathrm{mbm}}(t),
$$

where $\mathbb{J}_{s}(t)$ and $\mathbb{U}_{s}^{\mathrm{mbm}}(t)$ denote the existing and newly received global hypothesis sets of node $s$ at iteration $t=$ $0,1, \cdots$, respectively, $\mathbb{J}_{s}(0)$ denotes the initial global hypothesis set at node $s$ and $\mathbb{U}_{s}^{\mathrm{mbm}}(0) \triangleq \bigcup_{i \in \mathcal{S}_{s}} \mathbb{J}_{i}^{\mathrm{mbm}}$.

In flooding iteration $t=1,2, \cdots$, each local PMBM filter unionizes the new global hypotheses that its neighbors have received at the preceding iteration, i.e.,

$$
\mathbb{U}_{s}^{\operatorname{mbm}}(t)=\bigcup_{i \in \mathcal{S}_{s}}\left\{\mathbb{J}_{i}(t) \backslash \mathbb{J}_{i}(t-1)\right\},
$$

where $A \backslash B$ is the set difference of $A$ and $B$.

Let $\mathcal{S}_{s}^{[t]}$ denote the set of sensors that are at most $t$ hops away from sensor $s$, including sensor $s$ itself. Once flooding is completed at iteration $T$, sensor $i$ receives the hypotheses of all sensors $T$ hops away i.e.,

$$
\mathbb{J}_{s}(T)=\bigcup_{i \in \mathcal{S}_{s}^{[T]}} \mathbb{J}_{i} \triangleq \mathbb{J}_{\mathcal{S}_{s}^{[T]}} .
$$

Convergence of the flooding scheme has been addressed in [41]. When $t$ is larger than the diameter $D_{m}$ of the network, all sensors will have exactly the same information, i.e., $\forall s=$ $1,2, \cdots, S, \mathbb{J}_{s}\left(t \geq D_{m}\right)=\mathbb{J}_{1: S}$. 


\section{Exact MBM-AA Fusion}

Given a mixture of MBMs corresponding to $\mathbb{J}_{\mathcal{S}_{s}^{[T]}}$ as in (22) of each sensor $s$, all the (original) posterior information about the detected targets generated by sensors $\mathcal{S}_{s}^{[t]}$ are available to sensor $s$ now. Then, following the "best-fit-of-mixture" fusion principle, we need to find a MBM that best fits this mixture of MBMs so that each sensor maintains a PMBM conjugate prior while using the posterior information from sensors $\mathcal{S}_{s}^{[t]}$, for which we have the following key result.

Lemma 2. Given a mixture of MBMs with probability distributions, $f_{i}\left(\mathbf{X}^{\mathrm{mbm}}\right), i \in \mathcal{S}_{s}^{[t]}$, the MBM that fits them the best, in the sense of minimizing both the sum of 2-norm distances to these $M B M$ distributions and the sum of KLDs with relative to these MBM distributions, is their AA. That is,

$$
\begin{aligned}
f_{\mathrm{AA}}\left(\mathbf{X}^{\mathrm{mbm}}\right) & =\underset{g}{\arg \min } \sum_{i \in \mathcal{S}_{s}^{[t]}}\left\|f_{i}-g\right\|^{2}, \\
f_{\mathrm{AA}}\left(\mathbf{X}^{\mathrm{mbm}}\right) & =\underset{g}{\arg \min } \sum_{i \in \mathcal{S}_{s}^{[t]}} D_{\mathrm{KL}}\left(f_{i} \| g\right), \\
\text { s.t. } g & \propto \sum_{j \in \mathbb{J}} w_{j} \sum_{\mathbf{X}_{1}^{\mathrm{b}} \sqcup \ldots \sqcup \mathbf{X}_{n}^{\mathrm{b}}=\mathbf{X}^{\mathrm{mb}}} \prod_{i=1}^{n} f_{j, i}\left(\mathbf{X}^{\mathrm{b}}\right),
\end{aligned}
$$

Proof. The results (23) and (24) are the special MBM case of (11) and (12), respectively. The constraint (25) is automatically satisfied as proved in Lemma 1.

To perform the AA fusion, these hypotheses in $\mathbb{J}_{\mathcal{S}_{s}^{[T]}}$ need to be normalized. That is, $\forall j \in \mathbb{J}_{\mathcal{S}_{s}^{[T]}}$

$$
\bar{w}_{s, j}=\frac{w_{s, j}}{\sum_{j \in \mathbb{J}_{\mathcal{S}_{s}[T]}} w_{s, j}} .
$$

This leads to at sensor $s$ a mixture of re-weighted hypotheses/MBs collected from sensors $\mathcal{S}_{s}^{[t]}$,

$$
\left\{\bar{w}_{s, j},\left\{r_{\tilde{s}(j), j}^{(i)}, p_{\tilde{s}(j), j}^{(i)}(\cdot)\right\}_{i \in \mathbb{I}_{j}}\right\}_{j \in \mathbb{J}_{\mathcal{S}_{s}^{[T]}}},
$$

where $\tilde{s}(j)$ is the same as defined in (19).

Obviously, if $T \geq D_{m}$ the resulting MBM distribution is given in (16) otherwise the result is only an approximate. It is important to note that, these hypotheses are not independent but highly cross-correlated in a complicated manner. MBs from the same PMBM filter are more correlated with each other than than those from different PMBM filters. However, AA fusion has the advantage to deal with any degree of crosscorrelation for maintaining conservativeness [8], [9].

\section{E. Localization Accuracy}

While mixing the hypotheses from multiple sensors can compensate locally missed detections and significantly " $b i$ ased" tracks (in the sense that there is a large offset from the target position) due to model mismatching or unknown system input, it does not improve the localization accuracy of any particular BC corresponding to a potential target. Instead, the mixture gains conservativeness and robustness at the price of a larger distribution variance. Just like a trade-off is needed between localization error of the correctly detected targets and missed/false detection in the context of performance evaluation of multi-target trackers [6], [54]-[56], the fusion needs to trade off complete information for accurate estimation.

In realistic implementation, it is our observation that "partial consensus" (i.e., fusing only the key components of the posteriors that are more likely corresponding to targets [9][12], [14], [18]) turns out to be very useful albeit simply in reducing both the number of components and the variance of the mixture. More importantly, component merging and pruning and importance sampling have been demonstrated useful for improving the AA result to gain higher maximum a-posterior estimate accuracy [9]-[12], [14], [18]; see also [7]. To do so in the proposed MBM fusion, further fusion needs to be performed between $\mathrm{BCs}$ contained in hypotheses of distinct sensors. It is, however, intractable to associate the BCs corresponding to the same target from different hypotheses of distinct sensors and to accordingly merge them to get better localization accuracy. This requires "hypotheses merging" and is the key to improve the localization accuracy. We leave this to our future work.

\section{F. Communication and Computation Consideration}

To save communication, one may only communicate a few MBs of higher hypotheses weights $w_{s, j}, j \in \mathbb{J}_{s}$ in the MBM at each sensor $s$. This, referred to as partial consensus, has also been proved very necessary for PHD-AA fusion [11], [12], [14] and MB-AA fusion [9] and provides an effective strategy to combat the variance increase side-effect of the AA fusion [7]. For this purpose, there are two alternative types of thresholds. Specify a (maximum) number $N_{\mathrm{g}} \geq 1$ or (minimum) probability threshold $0<w_{\mathrm{g}}<1$, and only the $N_{\mathrm{g}}$ hypotheses with the largest target hypotheses probabilities from each MBM or the hypotheses with probability greater than $w_{\mathrm{g}}$ are disseminated and fused.

For computational efficiency, MBM may be approximated by a single MB distribution in a "best-fit-of-mixture" way [37], or simply by selecting a MB in the MBM that has the highest global hypothesis weight [39]. Then, the fusing of MB can be resorted to in the sense of AA fusion [9] or GA fusion [24]-[26], [39]. Obviously, MB is a special case of MBM when there is only one global hypothesis, namely $|\mathbb{J}|=1$. This avoids the non-closure problem of the MBM-GA fusion but seems unnecessary for the AA fusion which, as we have addressed, can fuse MBMs exactly and directly. So far, it remains unclear how to find a MBM to best approximate the GA of MBMs. In view of this, the AA fusion is advantageous over the GA fusion.

\section{Simulations}

We considered a planar space $[0,400] \times[0,400]$ which was monitored by a sensor network of diameter 4 consisting of 9 sensors as shown in Fig 4. The trajectories of totally five targets were generated using the method given in [29, Sec. VI] using the following target birth and dynamics models: The states of new born targets at time $k$ were a Poisson intensity 0.01 and an inaccurate Gaussian density with mean 


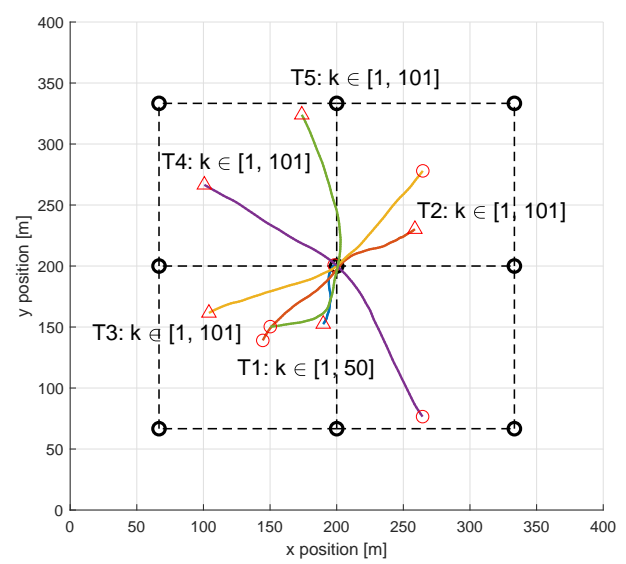

Fig. 4. Simulation scenario consisting of 9 sensors and 5 targets with various trajectories. The existence times $k$, starting positions (red triangles) and ending positions (red circles) of each target are shown.

$[200,0,200,0]^{\mathrm{T}}$ and covariance $\operatorname{diag}([200,2,200,2])^{2}$. Each target had a constant survival probability 0.95 . Denote by $\mathbf{x}_{k}=\left[p_{x, k}, \dot{p}_{x, k}, p_{y, k}, \dot{p}_{y, k}\right]^{\mathrm{T}}$ the target state consisting of position $\left[p_{x, k}, p_{y, k}\right]^{\mathrm{T}}$ and velocity $\left[\dot{p}_{x, k}, \dot{p}_{y, k}\right]^{\mathrm{T}}$, which followed a nearly constant velocity motion given as (with the sampling interval $\Delta=1$ s) transition density $f_{k \mid k-1}\left(\mathbf{x}_{k} \mid \mathbf{x}_{k-1}\right)=$ $\mathcal{N}\left(\mathbf{x}_{k} ; F \mathbf{x}_{k}, \mathbf{Q}\right)$ with

$$
F=\mathbf{I}_{2} \otimes\left[\begin{array}{cc}
1 & \Delta \\
0 & 1
\end{array}\right], \mathbf{Q}=0.01 \times \mathbf{I}_{2} \otimes\left[\begin{array}{cc}
\Delta^{3} / 3 & \Delta^{2} / 2 \\
\Delta^{2} / 2 & \Delta
\end{array}\right]
$$

where $\otimes$ is the Kronecker product.

Each sensor had the same time-constant target detection probability 0.8 and position measurement model as follows

$$
\mathbf{z}_{s, k}=\left[\begin{array}{cccc}
1 & 0 & 0 & 0 \\
0 & 0 & 1 & 0
\end{array}\right] \mathbf{x}_{k}+\left[\begin{array}{c}
v_{k, 1} \\
v_{k, 2}
\end{array}\right],
$$

with $v_{k, 1}$ and $v_{k, 2}$ as mutually independent zero-mean Gaussian noise with the same standard deviation of $1 \mathrm{~m}$.

In addition, clutter intensity is Poisson with rate $\kappa_{s}=10$ and uniformly distributed over the sensing field of each sensor $s=1,2, \cdots, 9$. The simulation was performed 100 runs with conditionally independent measurement series for each run.

The PMBM filter implementation used a maximum number of global hypotheses $N_{\max }=50$. Pruning was required for the maintenance of both PPP and MBM. Weight thresholds $10^{-2}$ and $10^{-3}$ were used for prune the low-weighted hypotheses and Poisson components, respectively. In the latter, BCs whose existence probability lower than $10^{-2}$ were removed. Ellipsoidal gating based on Mahalanobis distance with gate 4 was used for measurement-track association. For estimate extraction from the PMBM, the global hypothesis with highest weight was identified firstly. According to this hypothesis, each BC with existence probability larger than threshold 0.4 were used for extracting an target estimate by taking the mean of the BC, namely estimator 1 addressed in [36].

The filter performance is evaluated by the root mean square (RMS)-based generalized optimal subpattern assignment (GOSPA) error [56], which turns out to be a sum of localization errors for the properly detected targets and a cardinality error for missed and false targets, i.e., $d_{\text {RMS-GOSPA }}=$ $d_{\text {Loc }}+d_{\text {Card }}$ with localization error (Loc-Err) and cardinality error respectively defined as

$$
\begin{aligned}
d_{\text {Loc }}(|\hat{\mathbf{X}}|,|\mathbf{X}|) & =\min _{\pi \in \Pi_{|\hat{\mathbf{X}}|}} \sum_{i=1}^{|\mathbf{X}|} d^{(c)}\left(\mathbf{x}_{i}, \hat{\mathbf{x}}_{\pi(i)}\right)^{p}, \\
d_{\text {Card }}(|\hat{\mathbf{X}}|,|\mathbf{X}|) & =\frac{c^{p}}{2}(|\hat{\mathbf{X}}|-|\mathbf{X}|),
\end{aligned}
$$

if $|\hat{\mathbf{X}}| \geq|\mathbf{X}|$ otherwise $d_{\text {Loc }}(|\hat{\mathbf{X}}|,|\mathbf{X}|)=d_{\text {Loc }}(|\mathbf{X}|,|\hat{\mathbf{X}}|)$, $d_{\text {Card }}(|\hat{\mathbf{X}}|,|\mathbf{X}|)=d_{\text {Card }}(|\mathbf{X}|,|\hat{\mathbf{X}}|)$, where $\pi$ is a permutation on $\{1, \ldots, n\}, \Pi_{n}$ is the set of all permutations, and $d^{(c)}(\mathbf{x}, \mathbf{y})=$ $\min (d(\mathbf{x}, \mathbf{y}), c)$ is a metric between $\mathbf{x}$ and $\mathbf{y}$ cut-off at $c$.

We refer to the cardinality error as misdetection error (MDErr) and false-alarm error (FA-Err), respectively, depending whether the estimated number of targets is larger or smaller than the truth. We used $c=20, p=2$ in our simulation. Different numbers $T$ of flooding iterations from $T=0$ (without applying any information communication between sensors, namely noncooperative mode) to $T=4$ (the flooding algorithm is convergent) were applied. Further on, the disseminated number $N_{\mathrm{g}}$ of hypotheses of each local sensor was set as 1,2 and 5, respectively.

The RMS GOSPA error, Loc-Err, FA-Err and MD-Err of the PMBM filter at sensor 1 in the noncooperative mode or the fusion mode using parameters $T=1$ and $N_{\mathrm{g}}=1$ or 5 are given in Fig. 5. The mean errors of all PMBM filters at different sensors versus $T$ are given in Fig. 6, for $N_{\mathrm{g}}=$ 1,2 and 5, respectively. Furthermore, the mean communication cost (i.e., the number of real values be broadcasted by all sensors) and computing time required for each filtering step are given in Fig. 7. The results show:

1) The proposed MBM-AA fusion indeed improves the filters, significantly reducing the GOSPA error as compared with the non-cooperative filter. In particular, by applying flooding only for one iteration $(T=1)$ and only for disseminating a single hypothesis of each sensor $\left(N_{\mathrm{g}}=1\right)$ at each filtering step, the mean RMS GOSPA error can be significantly reduced as much as about $60 \%$. However, RMS-GOSPA/MD-error difference due to using different $N_{\mathrm{g}}$ and $T$ is non-obvious. This implies that more hypotheses do not contribute much new information. The RMS GOSPA reduction is mainly due to the reduction of the MD error as clearly shown in Fig. 5: they are almost equivalent and more significant than FA error and localization error. This indicates that misdetection forms a key challenge to the PMBM filter when the target detection probability is low.

2) The localization error and FA error have been increased by the proposed fusion as compared with the noncooperative mode. That is, the fusion does not make the filter get better localization accuracy for confirmed targets but may make the filter more prone to false alarm. Even worse, more flooding iterations cause larger FA and localization errors. The reasons could include: 1) fusion of a small number of (e.g., three) sensors is sufficient to largely compress the misdetection of a local sensor and more sensors do not help more; 2) the proposed MBM-AA fusion do not help improving 

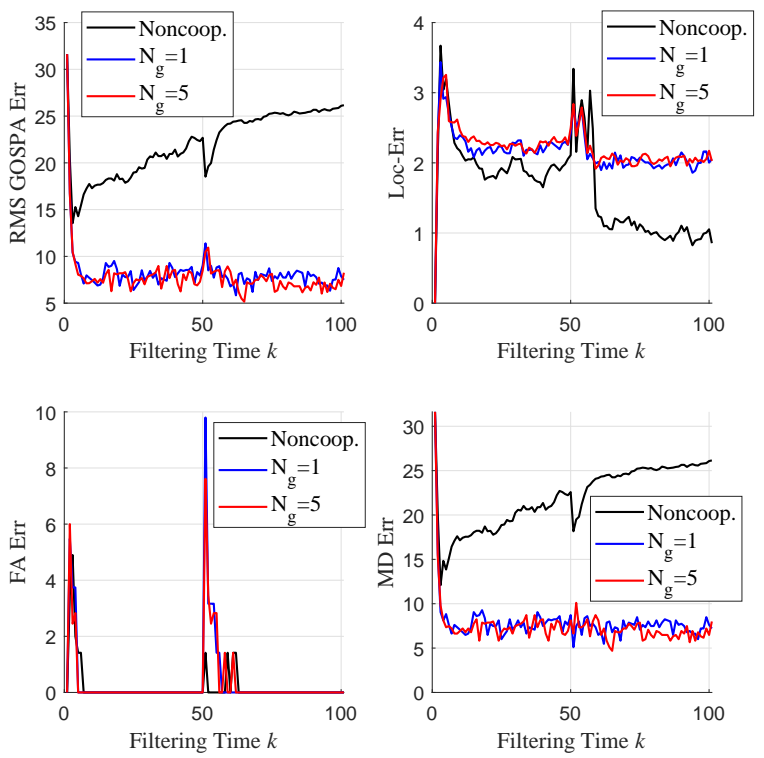

Fig. 5. RMS-GOSPA, Localization, FA, and MD errors of the PMBM filter at sensor 1 versus filtering steps in noncooperative mode and fusion mode using parameters $T=1$ and $N_{\mathrm{g}}=1$ or 5 , respectively.

the accuracy of any BCs including the significantlyweighted one that is used for estimate extraction; see our analysis in Section IV-E. Instead, it will reduce their weights in the fused mixture.

3) Communication cost and computing time required for fusion increase almost linearly with $T$ and $N_{g}$.

Overall, the simulation in this particular scenario demonstrates that it is sufficient to communicate only a single highest-weighted hypothesis among neighbor sensors. The gain is mainly from the reduced MD error, which is a key component of the GOSPA error of the filter with low target detection probability. It turns out to be unnecessary to apply the flooding information sharing for multiple iterations and multiple hypotheses, and for further than neighborhood. These findings are based on MBM-AA fusion without M$\mathrm{B} /$ hypotheses merging and Bernoulli merging/pruning in the fusion. Experimental studies in more complicated scenarios and when proper MB/Bernoulli merging/pruning is applied deserve to be investigated.

\section{CONCLUSION}

In this paper, we demonstrate that the linear, arithmetic average (AA) fusion essentially provides a theoretically best fit to the mixture of the fusing distributions which retains the complete information from these sources. The AA of multiple multi-Bernoulli mixtures (MBMs) remains a MBM and so it provides an exact, closed-form fusion solution for MBM fusion. This closure has been advocated for distributed PMBM filter design where the PMBM posterior is decomposed into PPP and MBM and only a part of MBMs are involved in the flooding communication and AA-fusion. Simulation based on a linear system has been given for demonstrating the performance of our approach including its strength and
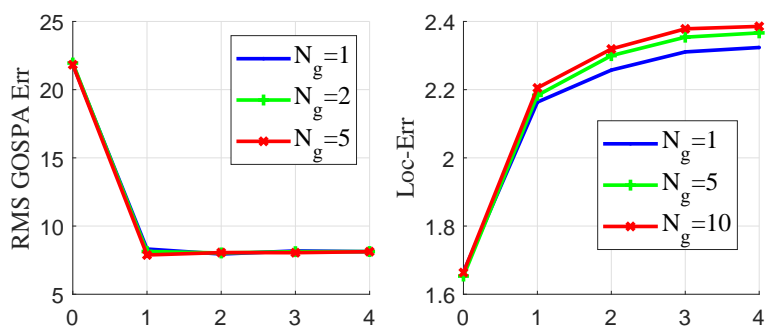

Number of Flooding Iterations $T$
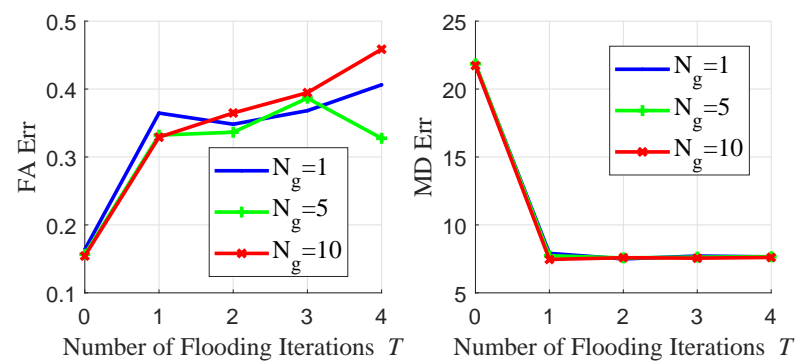

Fig. 6. Mean RMS-GOSPA, Localization, FA, and MD errors of all PMBM filters versus $T$ for different parameters $N_{\mathrm{g}}=1,2$ and 5 , respectively.

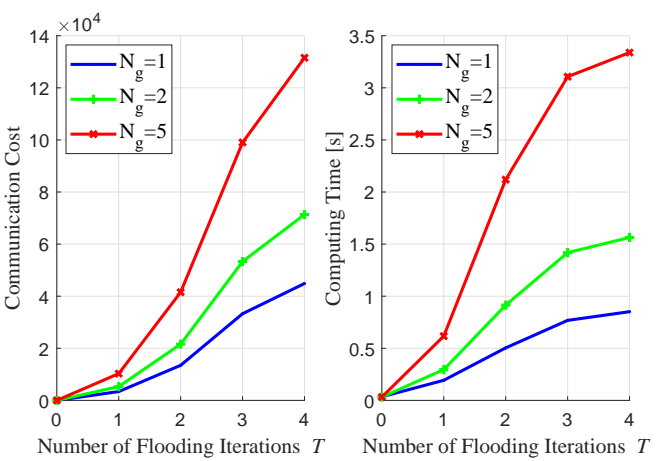

Fig. 7. Mean communication cost and computing time for each filtering step versus $T$, for $N_{\mathrm{g}}=1,2$ and 5 , respectively.

limitations. The proposed exact MBM-AA fusion approach benefits the filter significantly in combating misdetection but not in improving localization accuracy for which further fusion needs to be performed on the elemental Bernoulli components contained in hypotheses of distinct sensors. This forms our future research direction.

\section{REFERENCES}

[1] K. Chang, C. Y. Chong, and S. Mori, "Analytical and computational evaluation of scalable distributed fusion algorithms," IEEE Trans. Aerosp. Electron. Syst., vol. 46, no. 4, pp. 2022-2034, Oct. 2010.

[2] B.-N. Vo, M. Mallick, Y. Bar-shalom, S. Coraluppi, R. Osborne, R. Mahler, and B.-T. Vo, Multitarget Tracking. John Wiley \& Sons, Inc., 2015.

[3] F. Meyer, T. Kropfreiter, J. L. Williams, R. Lau, F. Hlawatsch, P. Braca, and M. Z. Win, "Message passing algorithms for scalable multitarget tracking," Proc. IEEE, vol. 106, no. 2, pp. 221-259, Feb. 2018.

[4] R. P. S. Mahler, Statistical Multisource-Multitarget Information Fusion. Norwood, MA, USA: Artech House, 2007.

[5] - Advances in Statistical Multisource-Multitarget Information Fusion. Norwood, MA, USA: Artech House, 2014.

[6] M. Beard, B. T. Vo, and B. N. Vo, "A solution for large-scale multiobject tracking," IEEE Trans. Signal Process., 2020, in press. 
[7] T. Li, H. Fan, J. García, and J. M. Corchado, "Second-order statistics analysis and comparison between arithmetic and geometric average fusion: Application to multi-sensor target tracking," Inf. Fusion, vol. 51, pp. $233-243,2019$.

[8] T. Bailey, S. Julier, and G. Agamennoni, "On conservative fusion of information with unknown non-Gaussian dependence," in Proc. FUSION 2012, Singapore, Jul. 2012, pp. 1876-1883.

[9] T. Li, X. Wang, Y. Liang, and Q. Pan, "On arithmetic average fusion and its application for distributed multi-Bernoulli multitarget tracking," IEEE Trans. Signal Process., 2020, in press.

[10] T. Li, J. Corchado, and S. Sun, "On generalized covariance intersection for distributed PHD filtering and a simple but better alternative," in Proc. FUSION 2017, Xi'an, China, Jul. 2017, pp. 808-815.

[11] _ "Partial consensus and conservative fusion of Gaussian mixtures for distributed PHD fusion," IEEE Trans. Aerosp. Electron. Syst., vol. 55, no. 5, pp. 2150-2163, Oct. 2019

[12] T. Li and F. Hlawatsch, "A distributed particle-PHD filter using arithmetic-average fusion of Gaussian mixture parameters," Inf. Fusion, Dec. 2018, under Review. Preprint: arXiv:1712.06128v2.

[13] A. K. Gostar, R. Hoseinnezhad, and A. Bab-Hadiashar, "CauchySchwarz divergence-based distributed fusion with Poisson random finite sets," in Proc. ICCAIS 2017, Chiang Mai, Thailand, Oct. 2017, pp. 112 116.

[14] T. Li, V. Elvira, H. Fan, and J. M. Corchado, "Local-diffusion-based distributed SMC-PHD filtering using sensors with limited sensing range,' IEEE Sensors J., vol. 19, no. 4, pp. 1580-1589, Feb. 2019.

[15] J. Y. Yu, M. Coates, and M. Rabbat, "Distributed multi-sensor CPHD filter using pairwise gossiping," in Proc. IEEE ICASSP 2016, Shanghai, China, Mar. 2016, pp. 3176-3180.

[16] L. Gao, G. Battistelli, and L. Chisci, "Multiobject fusion with minimum information loss," IEEE Signal Process. Lett., vol. 27, pp. 201 - 205, Jan. 2020.

[17] K. Da, T. Li, Y. Zhu, and Q. Fu, "Gaussian mixture particle jumpMarkov-CPHD fusion for multitarget tracking using sensors with limited views," IEEE Trans. Signal Inform. Process. Netw., Feb. 2020, in revision.

[18] T. Li, Z. Liu, and Q. Pan, "Distributed Bernoulli filtering for target detection and tracking based on arithmetic average fusion," IEEE Signal Process. Lett., vol. 26, no. 12, pp. 1812-1816, Dec. 2019.

[19] L. Gao, G. Battistelli, and L. Chisci, "Fusion of labeled RFS densities with minimum information loss," arXiv:1911.01083, Nov. 2019.

[20] R. P. S. Mahler, "Toward a theoretical foundation for distributed fusion," in Distributed Data Fusion for Network-Centric Operations, D. Hall, C.Y. Chong, J. Llinas, and M. Liggins, Eds. Boca Raton, FL, USA: CRC Press, 2012, pp. 199-224.

[21] D. Clark, S. Julier, R. Mahler, and B. Ristic, "Robust multi-object sensor fusion with unknown correlations," in Proc. SSPD 2010, London, UK, Sep. 2010.

[22] G. Battistelli, L. Chisci, C. Fantacci, A. Farina, and A. Graziano, "Consensus CPHD filter for distributed multitarget tracking," IEEE J. Sel. Topics Signal Process., vol. 7, no. 3, pp. 508-520, Jun. 2013.

[23] M. B. Guldogan, "Consensus Bernoulli filter for distributed detection and tracking using multi-static doppler shifts," IEEE Signal Process. Lett., vol. 21, no. 6, pp. 672-676, Jun. 2014

[24] B. Wang, W. Yi, S. Li, L. Kong, and X. Yang, "Distributed fusion with multi-Bernoulli filter based on generalized covariance intersection," in Proc. RadarCon 2015, 2015, pp. 958-962.

[25] S. Li, W. Yi, B. Wang, and L. Kong, "Computationally efficient distributed multi-sensor multi-Bernoulli filter," in Proc. FUSION 2018, Jul. 2018.

[26] W. Yi, S. Li, B. Wang, R. Hoseinnezhad, and L. Kong, "Computationally efficient distributed multi-sensor fusion with multi-Bernoulli filter," IEEE Trans. Signal Process., vol. 68, pp. 241-256, 2020.

[27] M. Üney, D. E. Clark, and S. J. Julier, "Distributed fusion of PHD filters via exponential mixture densities," IEEE J. Sel. Topics Signal Process., vol. 7, no. 3, pp. 521-531, Jun. 2013.

[28] M. Üney, J. Houssineau, E. Delande, S. J. Julier, and D. E. Clark, "Fusion of finite set distributions: Pointwise consistency and global cardinality," IEEE Trans. Aerosp. Electron. Syst., vol. 55, no. 6, pp. 2759-2773, Dec. 2019.

[29] J. L. Williams, "Marginal multi-Bernoulli filters: RFS derivation of MHT, JIPDA, and association-based MeMBer," IEEE Trans. Aerosp. Electron. Syst., vol. 51, no. 3, pp. 1664-1687, Jul. 2015.

[30] K. Granström, P. Willett, and Y. Bar-Shalom, "Approximate multihypothesis multi-Bernoulli multi-object filtering made multi-easy," IEEE Trans. Signal Process., vol. 64, no. 7, pp. 1784-1797, Apr. 2016.

[31] A. F. García-Fernández, J. L. Williams, K. Granström, and L. Svensson, "Poisson multi-Bernoulli mixture filter: Direct derivation and implemen- tation," IEEE Trans. Aerosp. Electron. Syst., vol. 54, no. 4, pp. $1883-$ 1901, 2018

[32] Y. Xia, K. Granström, L. Svensson, and A. F. García-Fernández, "Performance evaluation of multi-Bernoulli conjugate priors for multitarget filtering," in Proc. FUSION 2017, 2017, pp. 1-8.

[33] J. Smith, F. Particke, M. Hiller, and J. Thielecke, "Systematic analysis of the pmbm, phd, jpda and gnn multi-target tracking filters," in Proc. FUSION 2019, 2019, pp. 1-8.

[34] B. Vo and B. Vo, "Labeled random finite sets and multi-object conjugate priors," IEEE Trans. Signal Process., vol. 61, no. 13, pp. 3460-3475, Jul. 2013.

[35] B.-T. Vo, B.-N. Vo, and D. Phung, "Labeled random finite sets and the Bayes multi-target tracking filter," IEEE Trans. Signal Process., vol. 62 , no. 24, pp. 6554-6567, Dec. 2014.

[36] A. F. García-Fernández, Y. Xia, K. Granström, L. Svensson, and J. L. Williams, "Gaussian implementation of the multi-Bernoulli mixture filter," in Proc. FUSION 2019, 2019, pp. 1-8.

[37] J. L. Williams, "An efficient, variational approximation of the best fitting multi-Bernoulli filter," IEEE Trans. Signal Process., vol. 63, no. 1, pp. 258-273, 2015

[38] A. F. García-Fernández and S. Maskell, "Continuous-discrete multiple target filtering: PMBM, PHD and CPHD filter implementations," IEEE Trans. Signal Process., vol. 68, pp. 1300-1314, 2020.

[39] M. Fröhle, K. Granström, and H. Wymeersch, "Decentralized Poisson multi-Bernoulli filtering for extended target tracking," $\operatorname{arX}$ iv:1901.04518v1, 2019.

[40] M. Fröhle, C. Lindberg, K. Granström, and H. Wymeersch, "Multisensor Poisson multi-Bernoulli filter for joint target-sensor state tracking," IEEE Trans. Intell. Veh., vol. 4, no. 4, pp. 609-621, Dec. 2019.

[41] T. Li, J. Corchado, and J. Prieto, "Convergence of distributed flooding and its application for distributed Bayesian filtering," IEEE Trans. Signal Inf. Process. Netw., vol. 3, no. 3, pp. 580-591, Sep. 2017.

[42] B. Vo, B. Vo, and A. Cantoni, "The cardinality balanced multi-target multi-Bernoulli filter and its implementations," IEEE Trans. Signal Process., vol. 57, no. 2, pp. 409-423, Feb. 2009.

[43] C. Chong, S.Mori, and K. Chang, "Distributed multitarget multisensory tracking," in Multitarget Multisensor Tracking: Advanced applications, Y. Bar-Shalom, Ed. Artech House, 1990.

[44] Y. Bar-Shalom and L. Campo, "The effect of the common process noise on the two-sensor fused-track covariance," IEEE Trans. Aerosp. Electron. Syst., vol. AES-22, no. 6, pp. 803-805, Nov. 1986.

[45] C. Chong, S. Mori, and K. Chang, "Graphical models for nonlinear distributed estimation," in Proc. FUSION 2004, Stockholm, Sweden, Jun. 2004.

[46] S. J. Julier, "Estimating and exploiting the degree of independent information in distributed data fusion," in Proc. FUSION 2009, Jul. 2009, pp. 772-779

[47] Y. Gao, X. R. Li, and E. Song, "Robust linear estimation fusion with allowable unknown cross-covariance," IEEE Trans. Syst., Man, Cybern., Syst., vol. 46, no. 9, pp. 1314-1325, Sep. 2016.

[48] J. K. Uhlmann, "General data fusion for estimates with unknown cross covariances," in Proc.SPIE, vol. 2755, 1996, pp. 2755-2767.

[49] S. Julier and J. Uhlmann, "A non-divergent estimation algorithm in the presence of unknown correlations," in Proc. ACC 1997, New Mexico, USA, Jun. 1997, pp. 2369-2373.

[50] J. Ajgl and M. Simandl, "Conservativeness of estimates given by probability density functions: Formulation and aspects," Inf. Fusion, vol. 20, pp. 117-128, 2014

[51] H. G. Hoang, B.-N. Vo, B.-T. Vo, and R. Mahler, "The CauchySchwarz divergence for Poisson point processes," IEEE Trans. Inf. Theory, vol. 61, no. 8, pp. 4475-4485, Aug. 2015.

[52] K. Da, T. Li, Y. Zhu, H. Fan, and Q. Fu, "Kullback-Leibler averaging for multitarget density fusion," in Proc. DCAI 2019, Avila, Spain, Jun. 2019, pp. 253-261.

[53] A. F. García-Fernández and B. N. Vo, "Derivation of the PHD and CPHD filters based on direct Kullback-Leibler divergence minimization," IEEE Trans. Signal Process., vol. 63, no. 21, pp. 5812-5820, 2015.

[54] D. Schuhmacher, B.-T. Vo, and B.-N. Vo, "A consistent metric for performance evaluation of multi-object filters," IEEE Trans. Signal Process., vol. 56, no. 8, pp. 3447-3457, Aug. 2008

[55] M. Beard, B. T. Vo, and B. N. Vo, "OSPA(2): Using the OSPA metric to evaluate multi-target tracking performance," in Proc. ICCAIS 2017, 2017, pp. 86-91.

[56] A. S. Rahmathullah, A. F. García-Fernández, and L. Svensson, "Generalized optimal sub-pattern assignment metric," in 2017 20th International Conference on Information Fusion (Fusion), Jul. 2017, pp. 1-8. 